Gerlane Coelho Bernardo Guerra ${ }^{1}$

Artur Quintulano BezerRa da Silva ${ }^{2}$

LUDMILA BARROSO FRANÇA²

Patrícia Maria Carvalmo Assunção ${ }^{2}$

Rafael Xavier Cabral ${ }^{2}$

Aurigena Antunes de Araújo Ferreira ${ }^{1}$

\section{Utilização de medicamentos durante a gravidez na cidade de Natal, Rio Grande do Norte, Brasil}

\author{
Drug use during pregnancy in Natal, Brazil
}

Palavras-chave

Gravidez/efeitos de drogas Uso de medicamentos

Cuidado pré-natal

Saúde da mulher

Questionários

Keywords

Pregnancy/drug effects Drug utilization Prenatal care

Women's Health

Questionnaires

\section{Resumo}

OBJETIVO: estudar o uso de medicamentos por gestantes atendidas durante o pré-natal em unidades básicas do Sistema Único de Saúde (SUS) na cidade de Natal, rio Grande do Norte, Brasil. MÉTODOS: foram entrevistadas 610 grávidas, entre o primeiro e o terceiro trimestre de gestação, que compareceram para consulta pré-natal em unidades de saúde localizadas nos quatro distritos sanitários de Natal, entre maio e julho de 2006. Os dados foram coletados com entrevistas estruturadas, baseando-se em perguntas uso-orientadas e medicamento-orientadas. Os fármacos foram classificados de acordo com o Anatomical Therapeutic Chemical Classification System (ATC) e segundo critérios de risco para a gestação da Food and Drug Administration (FDA). Utilizou-se teste do $\chi^{2}$ para análise dos dados. RESULTADOS: eram utilizados 1.505 medicamentos, obtendo-se uma média de 2,4 drogas por mulher. $\bigcirc$ uso de pelo menos um fármaco na gravidez foi relatado por $86,6 \%$ das gestantes. As classes mais utilizadas foram os antianêmicos $135,6 \%$ dos medicamentos), analgésicos $(24,9 \%)$, drogas para distúrbios gastrintestinais $(9,1 \%)$ e vitaminas $(7 \%)$. De acordo com a classificação do FDA, dos medicamentos empregados 42,7\% pertencem a categoria $A$ de risco; $27,1 \%$ à categoria $B$, $29,3 \%$ à categoria $C ; 0,3$ à categoria $D$ e nenhum à categoria $X$. Foram usados, no primeiro trimestre da gestação, $43,6 \%$ dos fármacos. Observou-se maior uso de medicamentos quanto maior a escolaridade e a renda familiar da mulher. A automedicação ocorreu em 12,2\% dos medicamentos; esse índice foi maior no primeiro trimestre de gravidez e em gestantes de baixa escolaridade e multigestas. CONCLUSÕES: as gestantes de Natal estão sendo expostas a uma variedade de medicamentos, cuja segurança na gravidez ainda é incerta, o que exige prescrição criteriosa para evitar possíveis danos ao feto.

\section{Abstract}

PURPOSE: to study the use of medicines by pregnant women during prenatal care in clinics of the national public health system in the city of Natal, Brazil. METHODS: a total of 610 pregnant women between the first and the third trimesters of pregnancy were interviewed in the public clinics of the four sanitary districts of Natal, from May to July 2006. The data were collected by a structured questionnaire, based in use-oriented and medicine-oriented questions. The drugs were classified according to the Anatomical Therapeutic Chemical Classification System (ATC), in agreement with the gestation risk criteria from the Food and Drugs Administration (FDA). The statistical analysis was made by the $\chi^{2}$ test. RESULTS: a total of 1,505 drugs were used, with an average of 2.4 medications per woman. The use of at least one drug was found in $86.6 \%$ of the women. The most frequently used drugs were anti-anemics (35.6\%), analgesics (24.9\%), drugs for gastrointestinal disorders (9.1\%) and vitamins (7\%). According to the FDA classification, $42.7 \%$ belonged to category A risk, $27.1 \%$ to category B, $29.3 \%$ to category C, $0.3 \%$ to category D and none to category $X$. The use of medicines during the first trimester of pregnancy amounted to $43.6 \%$. The rate of drug use increased with higher schooling level and family income. Self-medication was found in $12.2 \%$ of the drug intake and this rate was higher in the first trimester of gestation and with women with low education level and previous gestations. CONCLUSIONS: pregnant women from Natal are being exposed to a variety of medicines with uncertain safety in pregnancy. Therefore, more careful prescription is needed, to avoid possible fetal damage.
Correspondência:

Ludmila Barroso France Rua Joũo Paulo I, 2.163 - Candeláría CEP 59064-470 - Natal/RN Fone: (84) 3234-5809 E-mail: Iudmilo@digi.com.br
Trabalho realizado no Departamento de Biofísica e Farmacologia da Universidade Federal do Rio Grande do Norte UFRN - Natal (RN), Brasil.

'Professora Adjunta de Farmacologia da Universidade Federal do Rio Grande do Norte - UFRN - Natal (RN), Brasil. ${ }^{2}$ Acadêmicos do Curso de Medicina da Universidade Federal do Rio Grande do Norte - UFRN - Natal (RN), Brasil. 


\section{Introdução}

O uso de medicamentos durante a gestação sempre representou um desafio para a classe médica, uma vez que implica ação potencialmente danosa não apenas para a mulher, mas também para o concepto. A maioria dos fármacos administrados a mulheres grávidas atravessa a barreira placentária e expõe o embrião em desenvolvimento a seus efeitos farmacológicos ${ }^{1}$. No entanto, os fármacos que são reconhecidamente teratogênicos representam um grupo bastante pequeno, englobando menos de 30 medicamentos ${ }^{2}$. Dessa forma, evitar completamente o uso de medicamentos é irreal e até danoso para mulheres portadoras de doenças crônicas ou que sofram intercorrências médicas durante a gestação. Deve-se, portanto, observar se os benefícios superam os possíveis riscos causados à mãe e ao feto quando há necessidade de prescrição de drogas durante a gravidez.

Diversos autores descreveram a prevalência do uso de medicações durante a gravidez, havendo variações nos métodos (medicamentos prescritos, dispensados em farmácias ou reportados em entrevistas) e nos critérios de inclusão de drogas. Em estudo realizado nos Estados Unidos, a taxa de uso de pelo menos um medicamento pela gestante foi de $56 \%$ (excluindo vitaminas e suplementos), com média de 2,2 fármacos por mulher ${ }^{3}$. Outros estudos que incluíam vitaminas e suplementos obtiveram taxas mais altas, como na Holanda $(79,1 \%)^{4}$, na Finlândia $(96,4 \%)^{5}$, na França $(99 \%)^{6}$ e nos Estados Unidos $(82 \% \text {, com média de } 2,7)^{7}$. Trabalhos nacionais mostraram variações entre 83,8 e $94,5 \% \%^{8-10}$ no uso de pelo menos um medicamento durante a gestação, incluindo vitaminas e suplementos.

As classes de fármacos mais comumente usados incluem preparados de ferro, ácido fólico, antibióticos, analgésicos, antiácidos, antieméticos e antiasmáticos, com pequenas diferenças entre os diversos estudos ${ }^{3,4,7,8}$.

A classificação dos medicamentos segundo critérios da agência norte-americana Food and Drug Administration (FDA) é constantemente abordada nas pesquisas, por avaliar o possível risco ao qual as gestantes estão sendo expostas. O percentual de medicamentos incluídos nas categorias $\mathrm{D}$ e X, consideradas potencialmente teratogênicas, varia de 1,1 a 3,9\% $\%^{3,4,7}$; no entanto, o número de gestantes em uso de medicamentos sobre os quais não há informação sobre segurança na gravidez chega a $79 \%^{6}$. Além disso, um estudo com mulheres não-grávidas mostrou que a prescrição de medicamentos de categorias D ou X em apenas metade dos casos era acompanhada de orientações quanto à contracepção ${ }^{11}$.

Quanto ao perfil das gestantes que mais usaram medicamentos, foi encontrada, na literatura, maior tendência de uso entre aquelas com menor escolaridade e maior número de filhos ${ }^{3}$. Outro aspecto associado à freqüência de utilização de medicamentos foi a assistência pré-natal, uma vez que o uso foi maior entre aquelas que iniciaram o pré-natal mais cedo (no primeiro trimestre) e que tiveram maior número de consultas ${ }^{10}$.

Excetuando-se alguns poucos trabalhos em cidades isoladas do Brasil, não há, na literatura, uma ampla abordagem sobre o tema na nossa realidade. Neste sentido, o trabalho se propôs avaliar o uso dos medicamentos por gestantes de um centro urbano do nordeste do Brasil, como é o caso de Natal, Rio Grande do Norte.

\section{Métodos}

A população do estudo foi composta por mulheres gestantes entre o primeiro e o terceiro trimestre de gravidez, que se apresentavam para consulta pré-natal pelo Sistema Único de Saúde (SUS) em uma das 13 unidades de saúde selecionadas por sorteio aleatório, na cidade de Natal, Rio Grande do Norte, Brasil.

A amostra de conveniência constituiu-se de gestantes da população de estudo, as quais compareceram ao atendimento médico durante o período de junho a agosto de 2006, totalizando 610 mulheres, distribuídas proporcionalmente pelos quatro distritos de saúde (Norte, 210; Sul, 140; Leste, 100; Oeste, 160). Essa amostra representou cerca de 2,5\% do total de grávidas acompanhadas anualmente pelo SUS de Natal, segundo dados do Sistema de Informação em Atenção Básica (SIAB), e admitiu-se erro de amostragem de $4 \%$. O estudo foi aprovado pelo Comitê de Ética em Pesquisa da Universidade Federal do Rio Grande do Norte (UFRN), segundo protocolo $\mathrm{n}^{\circ}$ 013/06, SISNEP 83067.

As variáveis englobadas no estudo foram: dependentes (uso de medicamentos, classe farmacológica, fonte de informação para seu uso - se prescrito ou não - e trimestre da gestação em que foi usado) e independentes (idade, escolaridade, estado conjugal, ocupação, renda familiar, gestações anteriores, tabagismo e doença crônica).

Foram realizadas entrevistas estruturadas com o preenchimento do formulário pelo pesquisador. $\mathrm{O}$ instrumento da pesquisa foi originalmente elaborado pelos pesquisadores e pré-testado em dez gestantes, selecionadas ao acaso, em unidade de saúde não englobada na pesquisa. A abordagem seguiu a linha de outros trabalhos semelhantes $^{7-9}$, em que foram feitas perguntas sobre $o$ uso de medicamentos para os diversos sinais e sintomas relacionados ou não à gravidez, como por exemplo: usou algum medicamento (qual) para febre cólica/enjôo etc.? Ainda foram feitas perguntas dirigidas para vitaminas, ferro e ácido fólico e para qualquer outro tipo de medicamento que a gestante recordasse. 
Os dados coletados foram armazenados e processados no programa Statistica, versão 5.5. Utilizou-se estatística descritiva e teste do $\chi^{2}$ para análise dos resultados, considerando-se significante um valor $\mathrm{p}<0,05$. As correlações testadas pelo $\chi^{2}$ foram as variáveis independentes (características das gestantes) com o uso de medicamentos (até dois ou mais de dois fármacos) e com o uso de medicamentos prescritos (até 50 ou mais de $50 \%$ do total de fármacos usados pela mulher).

As drogas foram divididas por classe terapêuticas de acordo com o Anatomical Therapeutic Chemical Classification System (ATC), da Organização Mundial da Saúde (OMS) ${ }^{12}$. Os medicamentos foram enquadrados ainda na classificação de risco para uso na gestação, segundo critérios da FDA, encontrada em Briggs et al. ${ }^{13}$. As categorias quanto ao risco são: A (ensaios controlados em humanos não demonstraram risco fetal), B (ensaios em animais indicaram ausência de riscos fetais, mas não há estudos controlados em humanos), C (ensaios em animais mostraram efeitos adversos, mas não há estudos controlados em humanos), D (evidência de risco fetal em humanos, mas benefícios podem se sobrepor aos riscos) e $\mathrm{X}$ (evidência de riscos fetais que não justificam quaisquer benefícios). Aqueles cuja classificação não foi encontrada na literatura foram agrupados na categoria $\mathrm{C}$, conforme orientação de Briggs et al. ${ }^{13}$. Os medicamentos constituídos de associações de mais de um fármaco foram considerados como da classe de maior risco apresentada por seus componentes.

Os fármacos citados que continham dois princípios ativos foram divididos em seus respectivos compostos químicos. As associações que continham mais de dois princípios ativos, assim como os medicamentos dos quais a mulher não recordava a denominação, foram realocados para a categoria outros.

O estudo não considerou como medicamento as suplementações alimentares, as vacinas, os compostos homeopáticos, bem como os chás e outras preparações fitoterápicas.

\section{Resultados}

Das 610 gestantes entrevistadas, 54,3\% correspondiam à faixa de 21 a 30 anos, $60,6 \%$ referiam terem até oito anos de estudo, 55,1\% referiam união consensual como estado civil, $68,2 \%$ não possuíam emprego remunerado, $57,7 \%$ relatavam renda familiar entre um e dois salários-mínimos e 10,5\% eram tabagistas. Em relação ao passado obstétrico, 39,2\% estavam na primeira gestação, $40,3 \%$ na segunda ou terceira e $20,5 \%$ na quarta ou mais. Estavam no primeiro trimestre da atual gestação $13,3 \%$, no segundo, 39,8\% e no terceiro, $46,9 \%$. O acompanhamento pré-natal foi referido como iniciado no primeiro trimestre da gestação por $50,3 \%$ das mulheres, no segundo por $45,6 \%$ e no terceiro por apenas $4,1 \%$. Eram portadoras de doença crônica $9,3 \%$, sendo as mais citadas: hipertensão arterial sistêmica $(40,4 \%)$, asma $(24,6 \%)$, diabetes mellitus $(10,5 \%)$, doenças da tireóide $(8,8 \%)$ e epilepsia $(5,3 \%)$.

Era utilizado um total de 1.505 medicamentos pelas gestantes, gerando média de 2,4 medicamentos (princípios ativos diferentes) per capita e mediana de 2 (intervalo de 0 a 9). $\mathrm{O}$ uso de pelo menos um medicamento durante a atual gravidez foi relatado por $86,6 \%$ das mulheres.

Como pode ser visto na Tabela 1, observou-se significância estatística na relação entre o uso de medicamentos e as seguintes variáveis: escolaridade (predominância do uso quanto maior o grau de instrução) e renda familiar (maior poder aquisitivo implicou maior uso). Dessa forma, observou-se que, entre as gestantes com menos de oito anos de estudo, $38,4 \%$ utilizaram mais de dois medicamentos, sendo que esse valor sobe para 52,5\% naquelas com mais de oito anos de estudo. $\mathrm{O}$ uso de mais de dois fármacos foi reportado por $53,3 \%$ das gestantes com renda superior a dois salários-mínimos e por apenas 35,7\% daquelas com renda de até um salário-mínimo. Não foi verificada diferença significante na relação entre o uso de medicamento e as variáveis faixa etária, estado civil e emprego remunerado. Nas correlações entre o uso de medicamentos e as variáveis tabagismo, doença crônica, número de gestações e trimestre de início do pré-natal, foram observadas diferenças significativas nas suas respectivas categorias. Para o passado obstétrico, não foi encontrado um padrão ordenado para o número de gestações, mas observou-se que o índice de gestantes que usaram mais de dois medicamentos foi maior para aquelas com mais de três gestações $(52,8 \%)$. Esse mesmo índice foi de $45,8 \%$ nas não-fumantes e de $28,1 \%$ nas fumantes, enquanto que entre as portadoras de doença crônica foi de $63,2 \%$ e naquelas sem doença foi de $42 \%$. A correlação de maior significância estatística foi observada em relação ao trimestre de início do pré-natal, uma vez que o uso de medicamentos durante a gravidez foi referido por $88,9 \%$ das mulheres que o fizeram no primeiro trimestre, por $85,6 \%$ das mulheres que iniciaram no segundo e por $68 \%$ daquelas que iniciaram no terceiro.

Os princípios ativos usados com maior freqüência foram: sulfato ferroso (22,7\% dos medicamentos), paracetamol $(13,1 \%)$, ácido fólico (12,9\%), dipirona $(10,4 \%)$, butilescopolamina $(8,3 \%)$ e dimenidrinato $(6,4 \%)$.

De acordo com o ATC, podemos afirmar que as classes terapêuticas mais utilizadas foram os antianêmicos - B03 (35,6\% dos medicamentos), seguido dos 
Tabela 1 - Distribuição dos medicamentos utilizados de acordo com as características socioeconômicas e de saúde das gestantes.

\begin{tabular}{|c|c|c|c|}
\hline \multirow{2}{*}{ Características } & \multicolumn{3}{|c|}{ Medicamentos usados } \\
\hline & De 0 a $2(\%)$ & Mais de $2(\%)$ & $p^{*}$ \\
\hline \multicolumn{4}{|l|}{ Grau de instrução } \\
\hline Até 8 anos de estudo & 61,6 & 38,4 & 0,0021 \\
\hline Mais de 8 anos de estudo & 47,5 & 52,5 & \\
\hline \multicolumn{4}{|l|}{ Renda familiar (S.M.) $)^{* *}$} \\
\hline Até 1 & 64,3 & 35,7 & 0,0098 \\
\hline Mais de 1 a 2 & 51,1 & 48,9 & \\
\hline Mais de 2 & 46,7 & 53,3 & \\
\hline \multicolumn{4}{|l|}{ Tabagismo } \\
\hline Sim & 71,9 & 28,1 & 0,0243 \\
\hline Não & 54,2 & 45,8 & \\
\hline \multicolumn{4}{|l|}{ Doença crônica } \\
\hline Sim & 36,8 & 63,2 & 0,0089 \\
\hline Não & 58,0 & 42,0 & \\
\hline \multicolumn{4}{|l|}{ Número de gestações } \\
\hline Apenas 1 & 54,4 & 45,6 & 0,0437 \\
\hline $2-3$ & 62,2 & 37,8 & \\
\hline Mais de 3 & 47,2 & 52,8 & \\
\hline \multicolumn{4}{|l|}{ Início do pré-natal } \\
\hline No primeiro trimestre & 48,5 & 51,5 & 0,0004 \\
\hline No segundo trimestre & 62,6 & 37,4 & \\
\hline No ferceiro trimestre & 76,0 & 24,0 & \\
\hline
\end{tabular}

*Foi utilizado teste do $\chi^{2}$ para obtenção do valor $p ;{ }^{*}$ *S.M.=salários-mínimos.

analgésicos - N02 (24,9\%), das drogas para distúrbios gastrintestinais - A03 $(9,1 \%)$ e das vitaminas - A11 $(7 \%)$, conforme - Figura 1.

A fonte de indicação das drogas ocorreu por prescrição médica em $87,8 \%$ das vezes, sendo, portanto, $12,2 \%$ por automedicação. Vale destacar o uso não prescrito do ácido acetilsalicílico por 18 gestantes, das 20 que o usaram, e da dipirona, por 64 das 157 que a usaram (Figura 2).

Observou-se diferença na relação entre o uso de medicamentos prescritos por médico e as variáveis: escolaridade $(\mathrm{p}=0,04)$, número de gestações $(\mathrm{p}=0,01)$ e trimestre atual da gravidez $(\mathrm{p}<0,05)$. Com isso, verificou-se que a taxa de automedicação foi maior no primeiro trimestre de gravidez e em gestantes de baixa escolaridade e multigestas.

Foi relatado uso durante o primeiro trimestre da gestação em 43,6\% dos medicamentos, sendo que, para o dimenidrinato, esse valor foi de $72,2 \%$; para o ácido acetilsalicílico foi de $70 \%$; e para o diclofenaco, $66,7 \%$ (Figura 3).

Em relação à classificação de risco na gravidez da FDA, obtivemos um total de 42,7\% na categoria A, 27,1\% na categoria $\mathrm{B}, 29,3 \%$ na categoria $\mathrm{C}, 0,3 \%$ na categoria $\mathrm{D}$ e nenhum na categoria $\mathrm{X}$ (Figura 4).

Das quatro grávidas que utilizaram medicamentos incluídos na categoria de risco $\mathrm{D}$, duas eram portadoras de

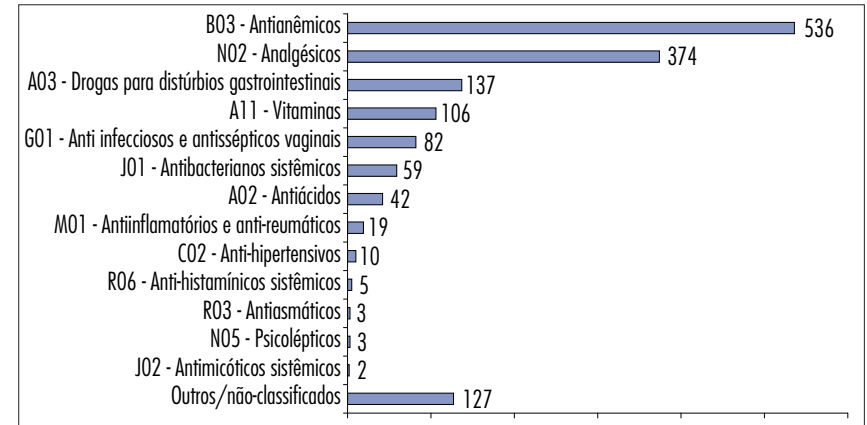

Figura 1 - Distribuição dos medicamentos utilizados segundo o Anatomical Therapeutic Chemical Classification System (ATC) da OMS ${ }^{12}$.

\begin{tabular}{|c|c|c|c|c|}
\hline & & $\begin{array}{l}\mathrm{N}^{0} \\
\text { Total }\end{array}$ & $\begin{array}{l}\% \\
\text { Prescrito }\end{array}$ & $\begin{array}{c}\% \mathbf{\square} \\
\text { Não Prescrito }\end{array}$ \\
\hline Sulfato ferroso & $\square$ & 342 & 98,5 & 1,5 \\
\hline Paracetamol & 1 & 197 & 76,1 & 23,9 \\
\hline Ácido fólico & 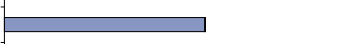 & 194 & 99,5 & 0,5 \\
\hline Dipirona & 1 & 157 & 59,2 & 40,8 \\
\hline Britilescopolamina & | & 125 & 89,6 & 10,4 \\
\hline Dimenidrinato $+B 6$ & 年 & 97 & 91,8 & 8,2 \\
\hline Nistatina & 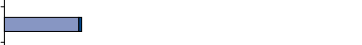 & 72 & 97,2 & 2,8 \\
\hline Associação vitamínica & | & 52 & 96,2 & 3,8 \\
\hline Cefalexina & 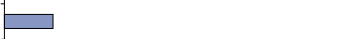 & 47 & 100 & 0 \\
\hline Hidróxido de Alumínio & 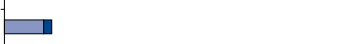 & 38 & 92,1 & 7,9 \\
\hline Vitamina $\mathrm{C}$ & 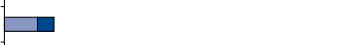 & 32 & 83,9 & 16,1 \\
\hline Complexo B- & & 22 & 100 & 0 \\
\hline AAS & 1 & 20 & 10,0 & 90,0 \\
\hline Diclofenaco & ש & 18 & 77,8 & 22,2 \\
\hline Metociopramida & & 11 & 100 & 0 \\
\hline Piperidolato/hesperidina & & 11 & 100 & 0 \\
\hline Metronidazol & & 10 & 100 & 0 \\
\hline Metildopa & & 10 & 100 & 0 \\
\hline Penicilina $G$ benzatina & & 6 & 100 & 0 \\
\hline Outros & 年 & 44 & 75,0 & 25,0 \\
\hline
\end{tabular}

Figura 2 - Número total dos medicamentos usados e percentual de prescrição.

\begin{tabular}{|c|c|c|c|c|}
\hline & & $\begin{array}{l}\mathrm{N}^{0} \\
\text { Total }\end{array}$ & $\begin{array}{l}\% \square \\
1^{\circ} \text { Trim. }\end{array}$ & $\begin{array}{l}\% \bar{\square} \\
\text { Outros Trim. }\end{array}$ \\
\hline Sulfato ferroso & E & 342 & 30,7 & 69,3 \\
\hline Paracetamol & 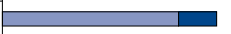 & 197 & 57,4 & 42,6 \\
\hline Ácido fólico & 1 & 194 & 45,9 & 54,1 \\
\hline Dipirona & 1 & 157 & 52,2 & 47,8 \\
\hline Brutilescopolamina & 1 & 125 & 46,4 & 53,6 \\
\hline Dimenidrinato + B6 & | & 97 & 72,2 & 27,8 \\
\hline Nistatina & | & 72 & 18,1 & 81,9 \\
\hline Associação vitamínica & \begin{tabular}{|l} 
\\
\end{tabular} & 52 & 34,6 & 65,4 \\
\hline Cefalexina & 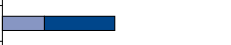 & 47 & 21,3 & 78,7 \\
\hline Hidróxido de alumínio & | & 38 & 23,7 & 76,3 \\
\hline Vitamina $C$ & 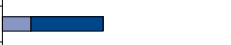 & 32 & 19,4 & 80,6 \\
\hline Complexo B & 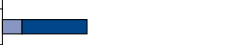 & 22 & 27,3 & 72,7 \\
\hline AAS & ש & 20 & 70,0 & 30,0 \\
\hline Didlofenaco & 年 & 18 & 66,7 & 33,3 \\
\hline Metoclopramida & 每 & 11 & 45,5 & 54,5 \\
\hline Piperidolato/hesperidina & 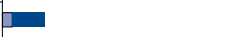 & 11 & 63,6 & 36,4 \\
\hline Metronidazol & 品 & 10 & 30,0 & 70,0 \\
\hline Metildopa & $\square$ & 10 & 80,0 & 20,0 \\
\hline Penicilina $G$ benzatina & 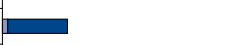 & 6 & 33,3 & 66,7 \\
\hline Outros & \begin{tabular}{|l} 
\\
\end{tabular} & 44 & 59,1 & 40,9 \\
\hline
\end{tabular}

Figura 3 - Número total de medicamentos usados e percentual de uso no primeiro trimestre de gestação. 


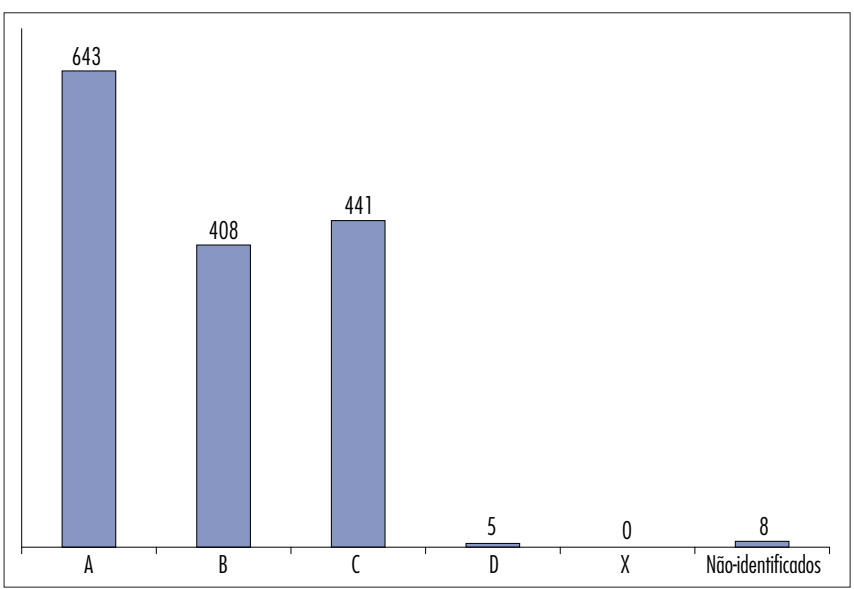

Figura 4 - Distribuição dos medicamentos utilizados segundo a classificação de risco da FDA.

epilepsia, sendo que uma fez uso de fenobarbital isolado e, a outra, associado à carbamazepina, ambos prescritos, por toda a gestação. As outras duas utilizaram diazepam, sendo um via intramuscular, no primeiro trimestre, e o outro via oral, automedicado, no terceiro trimestre.

Das entrevistadas, 38\% referiram uso de vacina (antitetânica) em algum momento da gravidez.

Acerca da orientação sobre o risco do uso de medicamentos durante a gravidez, 62,2\% das gestantes afirmaram já terem sido informadas a esse respeito por profissional de saúde.

\section{Discussão}

A pesquisa incluiu apenas gestantes atendidas pela rede pública de saúde, o que não nos permite generalizar os resultados obtidos para a população de Natal. Além disso, cabe a possibilidade de viés de aferição, uma vez que a utilização de entrevista como instrumento de coleta de dados está sujeita à memória, à confusão ou mesmo ao constrangimento das mulheres entrevistadas. No sentido de minimizar esse viés, foi realizado um estudo piloto, em que os entrevistadores simularam a aplicação do questionário em gestantes e, a partir disso, realizaram-se pequenas alterações no formulário, além de aprimoramentos na abordagem, visando a uma melhor compreensão das perguntas pelas entrevistadas.

Os valores obtidos no estudo sobre o uso de pelo menos um medicamento $(86,6 \%)$ foram um pouco inferiores, se comparados a alguns estudos europeus (96,4 a $99 \%)^{5,6}$ e do sul/sudeste do Brasil $(91,3 \text { a } 97,6 \%)^{8,10}$. Isso pode ter relação com o fato de o nosso estudo representar uma realidade nordestina, na qual predomina uma população de baixa renda e com maior dificuldade de acesso a medicamentos. Por outro lado, considerando-se que vários trabalhos internacionais baseiam-se em dados de prescrições médicas e que, especialmente na gravidez, os medicamentos prescritos nem sempre são usados, pode haver uma superestimação do uso. Em um estudo dinamarquês, encontrou-se que apenas $43 \%$ dos medicamentos prescritos eram de fato utilizados pelas gestantes ${ }^{14}$.

As classes de medicamentos mais usadas concordaram com os estudos nacionais, em que prevalecem os antianêmicos, os analgésicos, os fármacos que atuam sobre o aparelho gastrintestinal (antiespasmódicos e antieméticos) e os antiinfecciosos vaginais, havendo pequenas variações nas percentagens ${ }^{8-10}$. Em relação aos trabalhos internacionais s, $^{3,15}$, observou-se neles uma maior importância no uso de fármacos com ação sobre o aparelho respiratório e antibióticos sistêmicos do que neste estudo.

O acréscimo no uso de medicamentos com o aumento da escolaridade e da renda pode ser justificado como decorrente do maior esclarecimento sobre a necessidade do atendimento pré-natal. Além disso, a relação que o início do pré-natal guarda com o uso de medicamentos nos mostra o quanto o acompanhamento médico aumenta as médias de utilização, deixando claro o hábito de se prescrever a cada consulta.

A maioria das gestantes entrevistadas relatou já ter sido informada sobre o risco do uso de medicamentos na gravidez $(62,2 \%)$, ao contrário do que ocorreu no estudo de Fonseca et al. ${ }^{10}$, no qual esse número foi de apenas $27,7 \%$. Isso pode ter sido determinante para o baixo índice de automedicação encontrado nesta pesquisa $(12,2 \%)$.

Em relação à classificação de risco da FDA, obtevese que a maioria dos fármacos utilizados pertencia às categorias de risco A e B $(69,8 \%)$. Entretanto, pode ser considerado perigoso o fato de que quase $30 \%$ dos medicamentos pertenciam à categoria de risco $\mathrm{C}$, na qual a segurança é incerta por não haver estudos controlados em humanos.

Observou-se ainda que um grande percentual dos medicamentos $(43,6 \%)$ foi usado durante o primeiro trimestre gestacional, bem acima do valor encontrado em estudo brasileiro $(21,5 \%)^{10}$. Esse é um achado preocupante, tendo em vista que esse período é considerado o de maior risco de efeitos adversos fetais. No caso dos antiinflamatórios não-esteróides, cujo uso ocorreu predominantemente no primeiro trimestre da gravidez, é relatado aumento de até $80 \%$ no risco de aborto, sendo essa associação maior quando a utilização se faz próximo à concepção ou por mais de uma semana ${ }^{16}$. Além disso, os antiinflamatórios não-esteróides em doses antiinflamatórias são classificados na categoria de risco $\mathrm{D}$ durante o terceiro trimestre, não devendo ser usado após a $32^{\mathrm{a}}$ semana de gestação, pois têm sido associados à hipertensão pulmonar fetal, baixo peso ao nascer e alterações da coagulação. Nos casos de gestantes que necessitam de tratamento antiinflamatório, devemos dar preferência à prednisona em doses baixas. Para fins de analgesia, recomenda-se a substituição desse medicamento 
pelo paracetamol, fármaco classificado segundo a FDA na categoria B, sendo utilizado com segurança em todos os estágios da gravidez ${ }^{17}$.

Em relação ao uso disseminado do sulfato ferroso pelas gestantes, conforme visto na pesquisa, percebemos que isso reflete a rotina dos pré-natalistas da nossa região de prescrevê-lo não apenas como agente terapêutico, mas principalmente como profilático de anemia, o que, de certa forma, pode ser uma conduta questionável.

De acordo com os resultados obtidos, podemos inferir que o perfil das gestantes com maior tendência a usar medicamentos é composto por mulheres com mais de 30 anos, com renda familiar superior a dois saláriosmínimos, com mais de oito anos de estudo, casadas ou em união estável, empregadas, e que iniciaram o pré-natal no primeiro trimestre gestacional.

Está claro que as gestantes de Natal estão sendo expostas a uma variedade de medicamentos cuja segurança ainda não está estabelecida. Conseqüentemente, este uso deve ser melhor monitorado pelos médicos, os quais devem sempre buscar atualizações na literatura acerca dos possíveis riscos fetais ocasionados por cada fármaco.

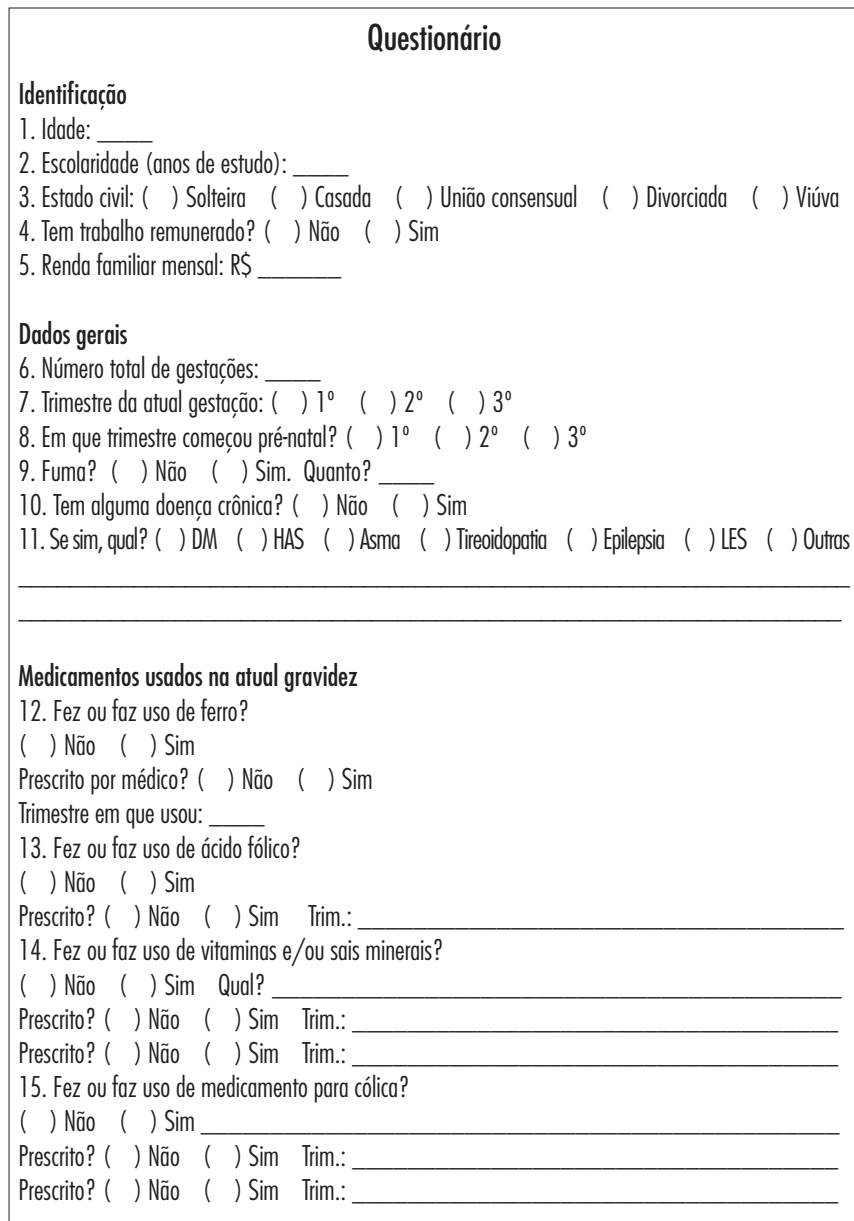

16. Fez ou faz uso de pomadas vaginais para corrimento?

( ) Não ( ) Sim

Prescrito? ( ) Não ( ) Sim Trim.:

Prescrito? ( ) Não ( ) Sim Trim.:

17. Fez ou faz uso de medicamento para dor ou febre?

( ) Não ( ) Sim

Prescrito? ( ) Não ( ) Sim Trim.:

Prescrito? ( ) Não ( ) Sim Trim.:

18. Fez ou faz uso de medicamento para azia?

( ) Não ( ) Sim

Prescrito? ( ) Não ( ) Sim Trim.:

Prescrito? ( ) Não ( ) Sim Trim.:

19. Fez ou faz uso de antibiótico?

( ) Não ( ) Sim

Prescrito? ( ) Não ( ) Sim Trim.:

Prescrito? ( ) Não ( ) Sim Trim.:

20. Fez ou faz uso de medicamentos para eniôo/vômitos?

( ) Não ( ) Sim

Prescrito? ( ) Não ( ) Sim Trim.:

Prescrito? ( ) Não ( ) Sim Trim.:

21. Fez ou faz uso de medicamentos para inflamação?

( ) Não ( ) Sim

Prescrito? ( ) Não ( ) Sim Trim.:

Prescrito? ( ) Não ( ) Sim Trim.:

22. Fez ou faz uso de outros medicamentos?

( ) Não ( ) Sim

Prescrito? ( ) Não ( ) Sim Trim.:

Prescrito? ( ) Não ( ) Sim Trim.:

Prescrito? ( ) Não ( ) Sim Trim.:

Prescrito? ( ) Não ( ) Sim Trim.:

Prescrito? ( ) Não ( ) Sim Trim.:

23. Fez ou faz uso de vacinas?

( ) Não ( ) Sim

24. Foi orientada por profissional de saúde sobre o risco de usar medicamentos durante a gravidez?

( ) Não ( ) Sim

\section{Referências}

1. Della-Giustina K, Chow G. Medications in pregnancy and lactation. Emerg Med Clin North Am. 2003;21(3):585-613.

2. Koren $G$, Pastuszak $A$, Ito $S$. Drugs in pregnancy. N Engl J Med. 1998;338(16): 1 128-37.

3. Riley EH, Fuentes-Afflick E, Jackson RA, Escobar G, Brawarsky $P$, Schreiber $M$, et al. Correlates of prescription drug use during pregnancy. J Womens Health (Larchmt). 2005; 14(5):401-9.
4. Bakker M, Jentink J, Vroom F, Van Den Berg P, De Walle H, De Jong-Van Den Berg LT. Drug prescription patterns before, during and after pregnancy for chronic, occasional and pregnancy-related drugs in the Netherlands. BJOG. 2006; 113(5):559-68.

5. Malm H, Martikainen J, Klaukka T, Neuvonen PJ; Finnish Register-Based Study. Prescription drugs during pregnancy and lactation-a Finnish register-based study. Eur J Clin Pharmacol. 2003;59(2): 127-33. 
6. Lacroix I, Damase-Michel C, Lapeyre-Mestre M, Montastruc JL. Prescription of drugs during pregnancy in France. Lancet. 2000;356(9243): 1735-6.

7. Andrade SE, Gurwitz JH, Davis RL, Chan KA, Finkelstein JA, Fortman K, et al. Prescription drug use in pregnancy. Am J Obstet Gynecol. 2004;191(2):398-407.

8. Mengue SS, Schenkel EP, Duncan BB, Schmidt MI. Uso de medicamentos por gestantes em seis cidades brasileiras. Rev Saúde Pública. 2001;35(5):415-20.

9. Osório-de-Castro CGS, Pepe VLE, Luiza VL, Cosendey MAE, Freitas AM, Miranda FF, et al. Uso indicado e uso referido de medicamentos durante a gravidez. Cad Saúde Pública. 2004;20 Supl 1: S73-82.

10. Fonseca MRCC, Fonseca E, Bergsten-Mendes G. Prevalência do uso de medicamentos na gravidez: uma abordagem farmacoepidemiológica. Rev Saúde Pública. 2002;36(2):205-12.

11. Schwarz EB, Postlethwaite DA, Hung YY, Armstrong MA. Documentation of contraception and pregnancy when prescribing potentially teratogenic medications for reproductive-age women. Ann Intern Med. 2007;147(6):370-6.
12. WHO Collaborating Centre for Drug Statistics Methodology. The Anatomical Therapeutic Chemical Classification System [text on the Internet]. Oslo: Norwegian Institute of Public Health; 1990 [cited 2006 Dec 12]. Available from: http:// www.whocc.no/atcddd

13. Briggs GG, Freeman RK, Yaffe SJ. Drugs in pregnancy and lactation. 7th ed. Baltimore: Williams \& Wilkins; 2005.

14. Olesen C, Søndergaard C, Thrane N, Nielsen GL, de Jongvan den Berg J, Olsen J; EuroMAP Group. Do pregnant women report use of dispensed medications? Epidemiology. $2001 ; 12(5): 497-501$.

15. Nordeng $H$, Eskild A, Nesheim BI, Jacobsen G. Drug use in pregnancy among parous Scandinavian women. Nor J Epidemiol. $2001 ; 11(1): 97-103$

16. Li DK, Liu L, Odouli R. Exposure to non-steroidal anti-inflammatory drugs during pregnancy and risk of miscarriage: population based cohort study. BM. 2003;327(7411):368.

17. Levy RA. O uso de drogas anti-reumáticas na gravidez. Rev Bras Reumatol. 2005;45(3):124-33. 